\title{
Successful Use Of Subcutaneous Infusion of Cortisol In An Adult Case Of Congenital Adrenal Hyperplasia
}

\author{
Dr Y. Mahgoub, Dr G. Cuthbert, Dr D. Kalathil, G. Morrison, Dr T. Purewal
}

Endocrinology \& Diabetes department, Royal Liverpool University Hospital, Liverpool, UK

\begin{abstract}
Introduction
Congenital adrenal hyperplasia $(\mathrm{CAH})$ is a group of a rare autosomal recessive disorders characterised by a deficiency of one of the enzymes necessary for cortisol biosynthesis. More than $90 \%$ of $\mathrm{CAH}$ is caused by mutations or deletions in cytochrome P450 21-hydroxylase gene. Impaired glucocorticoid synthesis results in chronic elevation of $\mathrm{ACTH}$ causing adrenal hyperplasia and accumulation of steroid precursors such as 17 hydroxyprogesterone (17OHP) (1). The main goal in CAH management is to replace deficient steroids in order to prevent adrenal crisis and to suppress the abnormal secretion of androgens.
\end{abstract}

\begin{abstract}
In addition to mineralocorticoid (fludrocortisone), different glucocorticoids can be used i.e.. prednisolone, dexamethasone or hydrocortisone twice or thrice a day is used. However, adequate and balanced replacement therapy with glucocorticoid is sometimes difficult to obtain. This is because of a number of factors such as patient tolerance, adverse effects and drugs pharmacokinetics and bioavailability (2).
\end{abstract}

\section{Clinical presentation \& results}

- In this case report, we present a 40 year old lady with a long standing history of congenital adrenal hyperplasia, which was not adequately controlled with conventional doses of oral glucocorticoid.

- With good compliance on hydrocortisone $15 \mathrm{mg}$ (morning) and $5 \mathrm{mg}$ (late afternoon) her average $17 \mathrm{OHP}$ was high in the morning at $21 \mathrm{nmol} / \mathrm{L}$ and during the day ranged between 2.9-4.9 nmol/L. Adjusting hydrocortisone doses and timings was not tolerated because of significant weight gain, stomach upset, anxiety and difficulty sleeping after the evening dose.

- Instead, prednisolone and dexamethasone (with different doses of $0.5 \mathrm{mg}-4 \mathrm{mg}$ twice a day) were tried but caused depression and intolerance. The $17 \mathrm{OHP}$ profile on dexamethasone was again significantly high in the morning at 47-56 $\mathrm{nmol} / \mathrm{L}$. The patient blamed the exacerbation of depression requiring hospital admission on dexamethasone and refused to take it again.

Management

- Using a continuous and variable subcutaneous hydrocortisone infusion (CSHI) via an insulin pump device, we achieved rapid control of her $\mathrm{CAH}$, attained normal cortisol circadian and $17 \mathrm{OHP}$ profiles and significantly improved her quality of life. Average daily hydrocortisone dose was $12-17.5 \mathrm{mg} /$ day, which produced on average 24-h serum cortisol and 17 OHP concentrations of 302.08 $\mathrm{nmol} / \mathrm{L}$ and $<2.3 \mathrm{nmol} / \mathrm{ml}$, respectively.

- The hydrocortisone basal rate via pump was adjusted to mimic the normal cortisol circadian rhythm (Fig.1).

- Quality of life was assessed on both $\mathrm{HC}$ tablets vs. CSHI, using standard SF-36 health survey. This showed a far better score with $\mathrm{CSHI}$ than $\mathrm{HC}$ tablets.

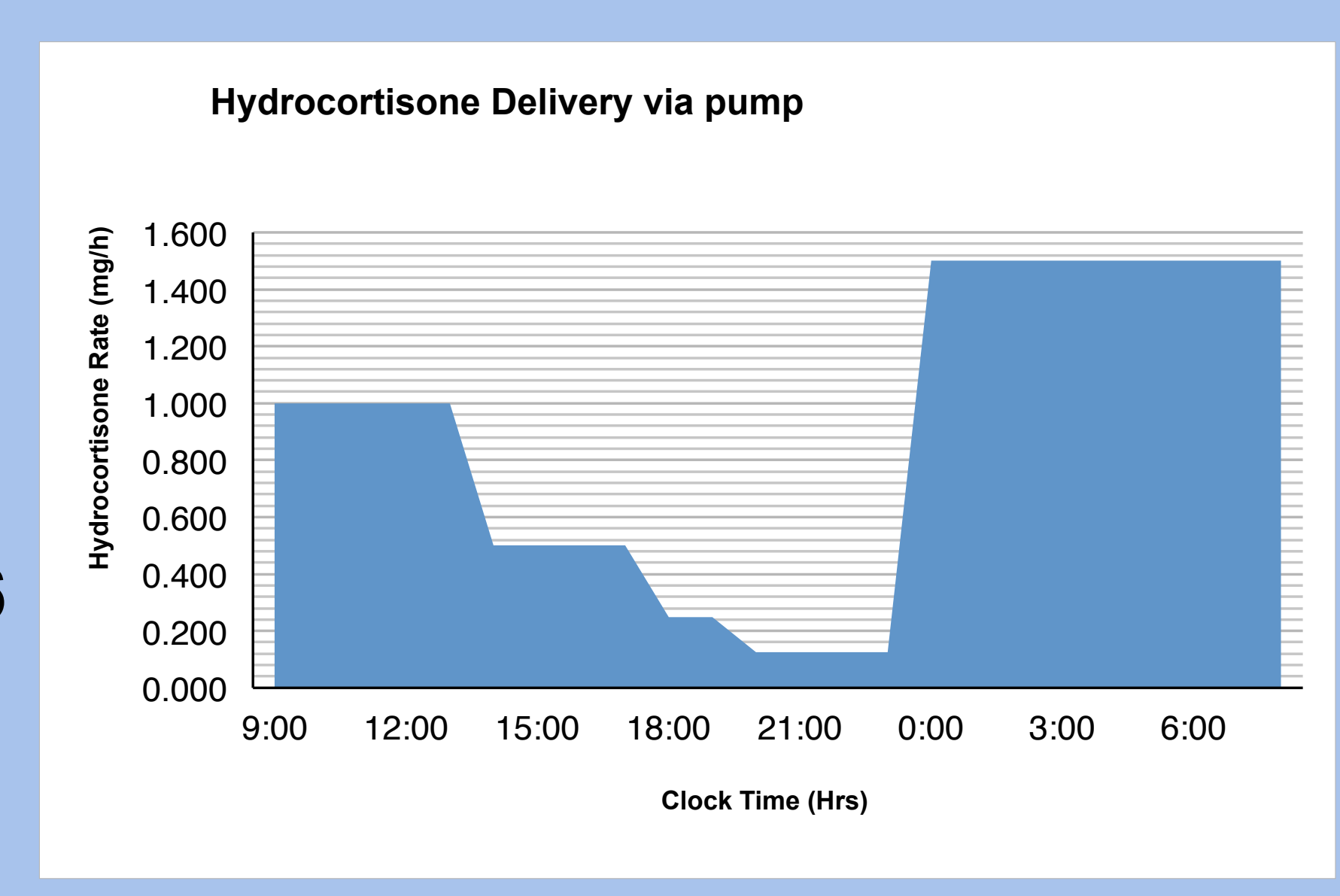

Fig. $1 \mathrm{HC}$ delivery via pump

\section{Discussion}

- Conventional oral hydrocortisone dosing does not mimic normal cortisol circadian rhythm. Various pharmacokinetic studies suggest a wide variation in cortisol bioavailability (range: $26-91 \%$ ). This is thought to be secondary to considerable individual variations in gut absorption effects, first-pass clearance at the liver, or both (Patel et al. 1984) (2). A number of factors influence the first-pass hepatic clearance such as gender, age, puberty and liver disease, because they affect the hepatic enzyme activity (3). In addition, some studies also suggest that oral hydrocortisone may result in supraphysiological cortisol levels, especially following the evening dose, due to decreased hydrocortisone clearance and increased bioavailability (4)

- In this case, $\mathrm{CSHI}$ produced rapid restoration of the normal cortisol circadian rhythmicity and optimised the 17 OHP day profile (Fig.2 \& Fig.3)

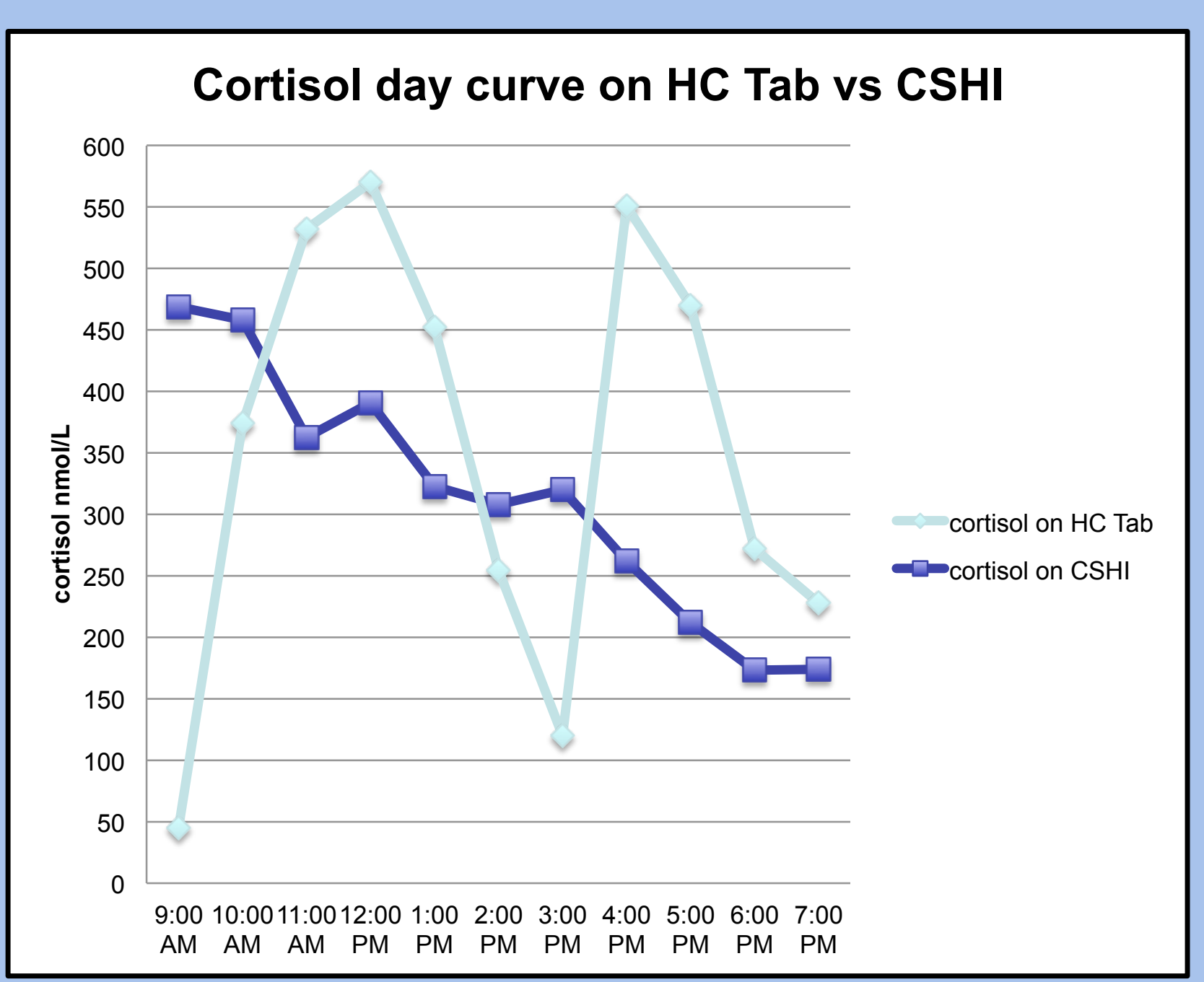

Fig.2: Cortisol day curve on $\mathrm{HC}$ Tab vs. CSHI

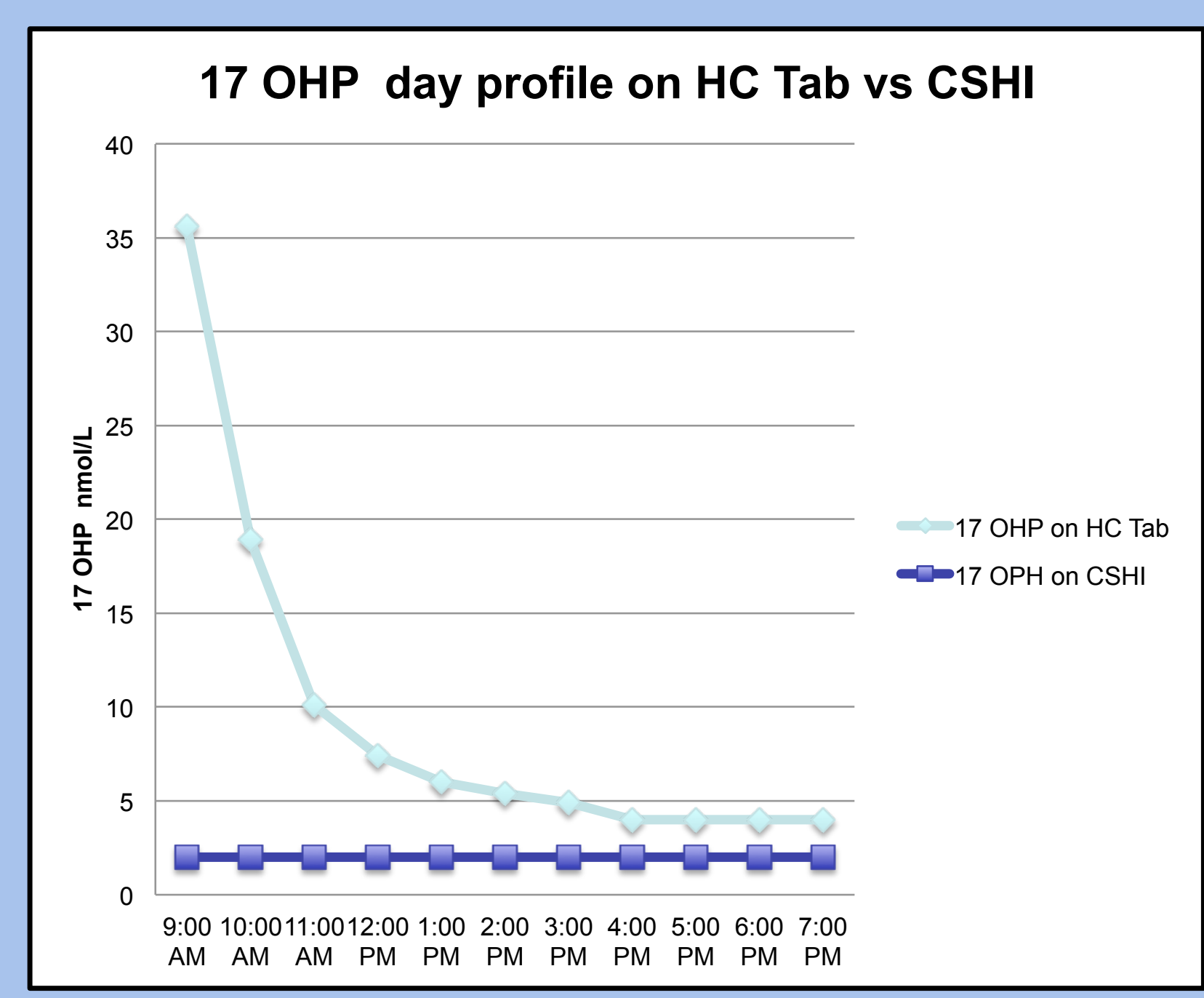

Fig.3: 17 OHP day profile on HC Tab vs. CSHI

\section{Conclusions}

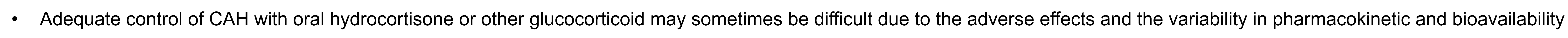
of the oral dose.

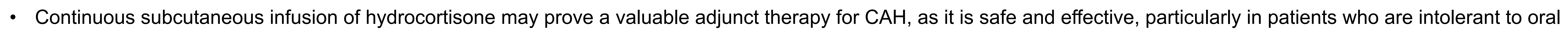
glucocorticoid and have variable drug pharmacokinetics and bioavailability.

\section{References}

1-White PC, Speiser PW 2000 Congenital adrenal hyperplasia due to 21-hydroxylase deficiency. Endocr Rev 21:245-291.

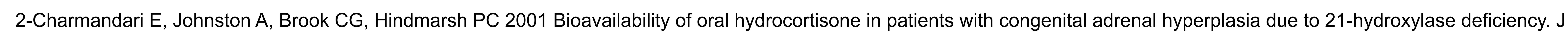
Endocr 169:65-70.

3-Løvås K, Husebye ES 2007 Continuous subcutaneous hydrocortisone infusion in Addison's disease. Eur J Endocrinol 157:109 -112

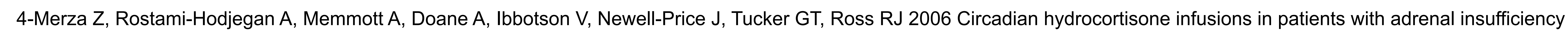
and congenital adrenal hyperplasia. Clin Endocrinol (Oxf) 65:45-50. 